\section{Optimization of Clarkson's Method for Calculating Absorbed Dose under Compensator Filters used in Intensity- modulated Radiation Therapy}

\author{
Pourkaveh M. ${ }^{1 \odot}$, Haghparast A.. ${ }^{2 *(\bullet)}$, Eivazi M. T. ${ }^{2}$, \\ Ghazikhanlu Sani K. ${ }^{3}$
}

\begin{abstract}
Background: Intensity Modulated Radiation Therapy (IMRT) is extensively used in the treatment of malignancies. Clarkson's method is one of the leading methods for dose calculation at open points present in irregular fields.

Objective: The aim of this study is to generalize the Clarkson's method for dose calculation at points under compensator filters in IMRT method and its application in IMRT quality control as well.

Material and Methods: In this experimental study, compensator filters were designed in two forms: flat filter and block piled-up compensator. The measurements for the compensator filters and open fields in 5 and $10 \mathrm{~cm}$ depths at energy levels (6, 10 and $18 \mathrm{MV}$ ) and in fields with different dimensions were performed using "Mapcheck2" dosimeter. The aim of performing calculations is to derive the theoretical dose by the generalized Clarkson's equation and comparing it with data resulted from the measurement for confirming the Clarkson's equation presented.
\end{abstract}

Results: These results demonstrate the data derived from the generalized Clarkson's method are in good agreement with the data resulted from measurement; the highest error of the proposed equation was 3\% for flat filter, and less than $5 \%$ for block-piled-up filter. Higher error in the block-piled-up filter compared with the flat filter was due to the presence of leakage between these blocks.

Conclusion: The results of this study demonstrated that the presented equation is reliable and valid, and the proposed equation can be applied for dose calculation at all points under the compensator filter or the shielded areas.

Citation: Pourkaveh M, Haghparast A, Eivazi MT, Ghazikhanlu Sani K. Optimization of Clarkson's Method for Calculating Absorbed Dose under Compensator Filters used in Intensity-modulated Radiation Therapy. J Biomed Phys Eng. 2020;10(5):575-582. doi: 10.31661/jbpe.v0i0.858.

\section{Keywords}

Radiotherapy, Intensity-Modulated; Compensator; Clarkson's Method; Absorbed Dose; Radiotherapy; Radiation Dosage

\section{Introduction}

Thtensity-modulated Radiation Therapy (IMRT) is extensively used in the treatment of malignancies. In this technique, several beams L with non-uniform intensity are used [1]. IMRT gives the ability to deliver a conformal dose distribution around the target, while lower doses to the neighboring critical organs enable tumor dose escalation [2-4]. IMRT with inverse planning system is carried out in 2 ways by applying either multi-leaf collimators (MLCs) or compensator filters
${ }^{1} \mathrm{MSc}$, Medical Physics

Department, Faculty of

Medicine, Kermanshah

University of Medical

Sciences, Kermanshah,

Iran

${ }^{2}$ PhD, Medical Physics

Department, Faculty of

Medicine, Kermanshah

University of Medical

Sciences, Kermanshah,

Iran

${ }^{3} \mathrm{PhD}$, Radiology De-

partment, Paramedi-

cal School, Hamadan

University of Medical

Sciences, Hamadan, Iran

*Corresponding author:

A. Haghparast

Medical Physics De-

partment, Faculty of

Medicine, Kermanshah

University of Medical

Sciences, Kermanshah, Iran

E-mail: a.haghparast@

kums.ac.ir

Received: 28 October 2017

Accepted: 4 April 2018 
[5-7]. Nowadays, compensator-based IMRTs are formed for attenuating photon flux in open fields, such that the passing intensity plan takes the form of improved dose flux [8-10]. The compensator technique has multiple advantages and disadvantages in comparison with the MLC technique [1]. Intensity modulation can be performed with higher resolution and smoother dose distribution in consequence of which problems originate from patient movement. Furthermore, the monitor unit (MU) can be reduced because multiple segments are unnecessary [7]; whereas, MLC systems have limitations in spatial resolution or segmental dimensions because of the increased number of incident monitor units (MU) or increased radiation time. There is also the possibility of patient's movement during treatment $[11,12]$. Moreover, because the compensator is static, its quality assurance (QA) stages are much more convenient than that of MLC $[9,10]$. In addition, the leakage radiation is higher than that of compensators and other circumstances $[13,14]$. Production of a compensator for each patient and field is a time-consuming task; however, outside supply and delivery systems are available [5]. Therefore, compensator IMRT has become a realistic possibility.

Practically, it is impossible to achieve 3D dose distribution delivered to the patient by use of direct measurement; therefore, treatment planning has been used based on calculation models [15]. In radiation therapy, dose calculations are obtained using treatment planning software, and are usually verified by the use of an independent computerized monitor unit calculation routinely or by applying manual calculations. Unlike conventional conformal therapy, the fields formed by use of MLC and compensator in IMRT are provided by inverse planning which due to their complexity, makes manual calculations for dose verification unfeasible [16]. One of the methods used for dose calculation at the points along the central axis and the off-axis is Clarkson's method. This method is used for only open areas in irregular fields that part of which has been shielded [17]; it is not usable for areas under compensator filters. One of the disadvantages of Clarkson's method is producing the scattered rays "exception of the areas shielded by the block and collimator" in the entire field uniformly. Factors such as the presence of wedge or non-uniformity of surface are disregarded [18]. Since quality assurance is used in IMRT, it is recommended that the verification of plan, pre-treatment measurements and periodic tests of the system are implemented. This method involves some difficulties such as long time needed for the performance of quality assurance test. Therefore, it is important to have an independent and reliable method for evaluating dose in IMRT plan.

The aim of this study is to derive the theoretical dose by using the generalized Clarkson's equation and comparing it with data resulted from the measurement (measured dose) for confirming the Clarkson's equation presented.

\section{Material and Methods}

Method of Obtaining Generalized Clarkson's Equation

In this experimental study, Clarkson's method is usually implemented for point dose calculation in fields with irregular forms when radiation-sensitive structures are shielded against primary beam or when the field extends beyond the irregularly shaped patient's body contour. Clarkson's method is based on the principle that the scattered component of the depth dose can be calculated separately from the primary component, which is independent of the field size and shape. For each irregular field, scattered dose at each point (Q) is calculated by dividing the field into equal sectors of $\overline{\Delta \theta}$ degrees. The scatter maximum ratio (SMR) values are calculated for sectors using SMR table for circular fields, and summed to give the average scatter maximum ratio $(\overline{\mathrm{SMR}})$ for irregular field according to 
equation (1) at Point Q.

$$
\overline{\operatorname{SMR}}\left(\mathrm{d}, \mathrm{r}_{\mathrm{d}}\right)=\frac{1}{n} \sum_{i=1}^{n} \operatorname{SMR}\left(d, r_{i}\right)
$$

Where $r_{i}$ is the radius of the $i$-th sector at depth $\mathrm{d}$, and $\mathrm{n}$ is the total number of sectors $(n=2 \pi / \Delta \theta)$. The computed $\overline{\operatorname{SMR}}\left(d, r_{d}\right)$ is then converted to average tissue maximum ratio $\overline{\operatorname{TMR}}\left(\mathrm{d}, \mathrm{r}_{\mathrm{d}}\right)$ by using equation (2):

$$
\overline{T M R}\left(d, r_{d}\right)=\left[\operatorname{TMR}(d, 0)+\overline{\operatorname{SMR}}\left(d, r_{d}\right)\right] \times \frac{S_{P}(0)}{\overline{S_{P}}\left(r_{d}\right)}
$$

Where, $\overline{\mathrm{S}_{\mathrm{p}}}\left(\mathrm{r}_{\mathrm{d}}\right)$ is the average phantom scatter factor for the irregular field and $S_{p}(0)$ is the phantom scatter factor for the $0 \times 0$ area field. TMR $(\mathrm{d}, 0)$ is the tissue maximum ratio for the $0 \times 0$ area field. The above equation is strictly valid only for points along the central axis of a beam that is normally incident on an infinite phantom with flat surface. $\overline{T M R}\left(d, r_{d}\right)$ is converted into percent depth dose by using equation (3):

$$
P D D=100 \times\left(\frac{f+t_{0}}{f+d}\right)^{2} \times \overline{T M R}\left(d, r_{d}\right) \times \frac{\overline{S_{P}}\left(r_{d}\right)}{\overline{S_{P}}\left(r_{t_{0}}\right)}
$$

Where, PDD is the percent depth dose. $\mathrm{f}$ is the source to surface distance. $d$ is the depth of measurement. $t_{0}$ is the reference depth of maximum dose. $\overline{S_{P}}\left(r_{t_{0}}\right)$ is the average phantom scatter factor for field size at reference depth [17].

In the common Clarkson's equation, TMR $(\mathrm{d}, 0)$ is defined as follows:

$$
\operatorname{TMR}(d, 0)=e^{-\mu\left(d-t_{0}\right)}
$$

In this equation, $d$ is the water depth and $\mu$ is the linear attenuation coefficient of water. However, when the compensator filter is put in the direction of radiation, $\operatorname{TMR}(\mathrm{d}, 0)$ shall be defined as $\operatorname{TMR}^{\prime}(d, 0)$ :

$$
\operatorname{TMR}^{\prime}(d, 0)=e^{-\mu\left(d-t_{0}\right)} \times e^{-\mu^{\prime} \chi}
$$

Where, $x$ is the compensator thickness, $\mu^{\prime}$ is the linear attenuation coefficient of the com- pensator. In addition to this, $e^{-\mu^{\prime} x}$ is defined as follows:

$$
e^{-\mu^{\prime} \chi}=\frac{D(d)}{D_{0}(d)}=T
$$

Where, $D_{0}(d)$ is the dose in open field at depth $d$ and $D(d)$ is the dose in the presence of filter at the same depth $\mathrm{d}$, $\mathrm{T}$ is the transmission factor. Therefore, putting equations (4), (5) and (6) together, we shall have:

$$
T M R^{\prime}(d, 0)=T \times T M R(d, 0)
$$

In the conventional Clarkson's equation, SMR value at points fully shielded is zero and, at points present in open fields, the concerned value is one. Therefore, in the presence of a compensator filter, we can use coefficient (2T1) "by using dosimeter, the doses of the various points of the field were measured for open fields in the absence of compensator filter and also in the presence of the filter, this way, the $\mathrm{D} / \mathrm{D}_{0}$ ratio, which is equal to $\mathrm{T}$, was derived for all of the points of the field" demonstrating compensator filter effect. Consequently, the general Clarkson's equation for any point of the field shall change as Generalized Clarkson's equation (8):

$\overline{T M R}\left(d, r_{d}\right)=\left[K_{P} \times T \times T M R(d, 0)+(2 T-1) \times \overline{\operatorname{SMR}}\left(d, r_{d}\right)\right] \times \frac{S_{P}(0)}{S_{P}\left(r_{d}\right)}(8)$

$\mathrm{K}_{\mathrm{p}}$ in this equation is the off-axis ratio representing primary dose at interest point relative to that of the central axis. $\overline{\operatorname{SMR}}\left(\mathrm{d}, \mathrm{r}_{\mathrm{d}}\right)$ in the above equation is achived by use of equation (1).

For deriving $\operatorname{SMR}\left(\mathrm{d}, r_{\mathrm{i}}\right)$ in equation(1), the Clarkson's drawing method (Figure 1) for the central point and the off-axis points regarding any of the fields of intensity plan with different dimensions at any of the three energy levels and the concerned depths are drawn. For each point in intensity plan of concerned field, sectors are drawn according to Clarkson's method with 10 degrees, then the lengths of each sector present in any pixel from the center to the 


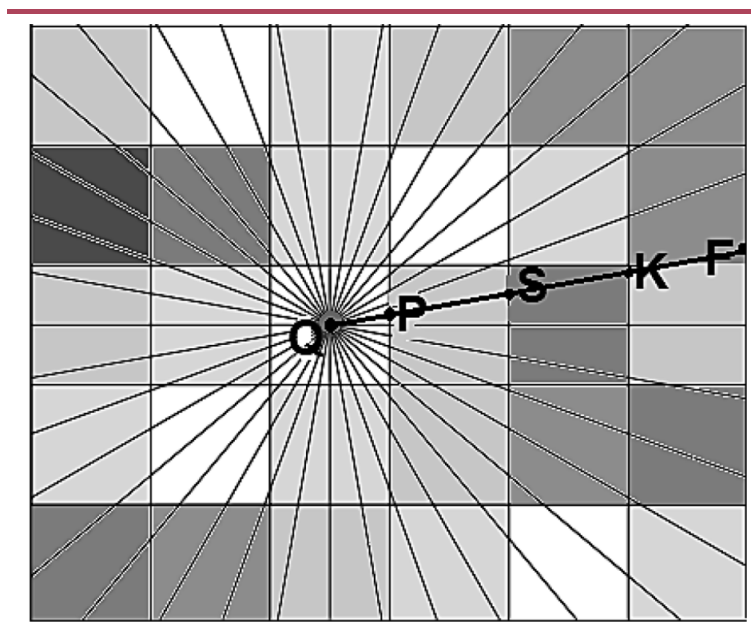

Figure 1: Clarkson's drawing for the points existing in the field.

end of any of the pixels were measured and, by the use of SMR table values, the SMR values relevant to these lengths were obtained. In each sector, for deriving total SMR of any sector equation (9) was applied.

Total SMR for a sector $(Q F)=$

$\sum\left(2 T_{Q P}-1\right) \times\left(S M R_{Q P}\right)+\left(2 T_{P S}-1\right) \times\left(S M R_{Q S}-S M R_{Q P}\right)+$

$\left(2 T_{S K}-1\right) \times\left(S M R_{Q K}-S M R_{Q S}\right)+\left(2 T_{K F}-1\right) \times\left(S M R_{Q F}-S M R_{Q K}\right)$

$\mathrm{T}$ Coefficient is related to each pixel of the fields. Afterwards, the above equation was applied for all sectors, $\overline{\operatorname{SMR}}\left(\mathrm{d}, \mathrm{r}_{\mathrm{d}}\right)$ was calculated by the use of equation (1) and was converted to $\overline{T M R}\left(d, r_{d}\right)$ by putting it in equation (8)

Then, by use of equation (3), $\overline{T M R}\left(d, r_{d}\right)$ was converted to PDD. Here, the theoretical dose is calculated by putting the calculated parameters in the below equation.

$$
M U=\frac{D_{\text {Teorical }} \times 100}{P D D \times S_{C, P} \times S S D \text { factor } \times K_{P} \times \text { output factor }}
$$

In the equation (10), SSD Factor is deduced as follows:

$$
\text { SSDfactor }=\left(\frac{S C D}{S S D+t_{0}}\right)^{2}
$$

Where, SCD is the source calibration distance.

Using equation (12), the difference percent between theoretical and measured doses can be measured.

$$
\text { Percent error }=\frac{\left|D_{\text {Measured }}-D_{\text {Teorical }}\right|}{D_{\text {Measured }}} \times 100(
$$

\section{Data Collection}

The measurements conditions

photon beams of 6,10 and $18 \mathrm{MV}$ produced by linear accelerator system (Elekta [SL75/25]) manufactured in England, depths of 5 and $10 \mathrm{~cm}$, Output Factor $=1 \mathrm{MU} / \mathrm{cGy}$ in maximum depth dose, 100 monitor unit (MU), Source Surface Distance $(\mathrm{SSD})=100 \mathrm{~cm}$, field sizes with dimensions of $4 \times 4 \mathrm{~cm}^{2}, 10 \times 10 \mathrm{~cm}^{2}$, $15 \times 15 \mathrm{~cm}^{2}$ and $20 \times 20 \mathrm{~cm}^{2}$ were used.

TMR and PDD values were obtained by the use of diode dosimeter and RFA300 phantom both manufactured by Scanditronix-Wellhofer of Germany-Sweden.

SMR values for field sizes with different dimensions and existing energies were obtained using equation (13):

$\operatorname{SMR}\left(d, r_{d}\right)=\operatorname{TMR}\left(d, r_{d}\right) \times\left(\frac{S_{P}\left(r_{d}\right)}{S_{P}(0)}\right)-\operatorname{TMR}(d, 0)(13)$

\section{Data Collection for Compensator} Filters

The compensator filters were designed in two forms: flat filter and block-piled-up. For making flat filters, brass plates with 1, 3 and 5 $\mathrm{cm}$ thicknesses were provided and these plates were used as filters against radiation. For block-piled-up compensators, cubic blocks made of brass with dimension $1 \times 1 \mathrm{~cm}^{3}$ were arranged for making stepped wedge filters (Figure 2) and Perspex blocks with dimension $1 \times 1 \mathrm{~cm}^{3}$ were used for filling the empty spaces inside the compensator box made of aluminum. The measurements were performed for the flat filter and open fields in 5 and $10 \mathrm{~cm}$ depths at energy levels of 6, 10 and $18 \mathrm{MV}$, and in fields dimensions of $4 \times 4 \mathrm{~cm}^{2}, 10 \times 10$ $\mathrm{cm}^{2}, 15 \times 15 \mathrm{~cm}^{2}$ and $20 \times 20 \mathrm{~cm}^{2}$, and at 1,3 and $5 \mathrm{~cm}$ thicknesses by the use of Mapcheck2 
Absorbed Dose under Compensator Filters

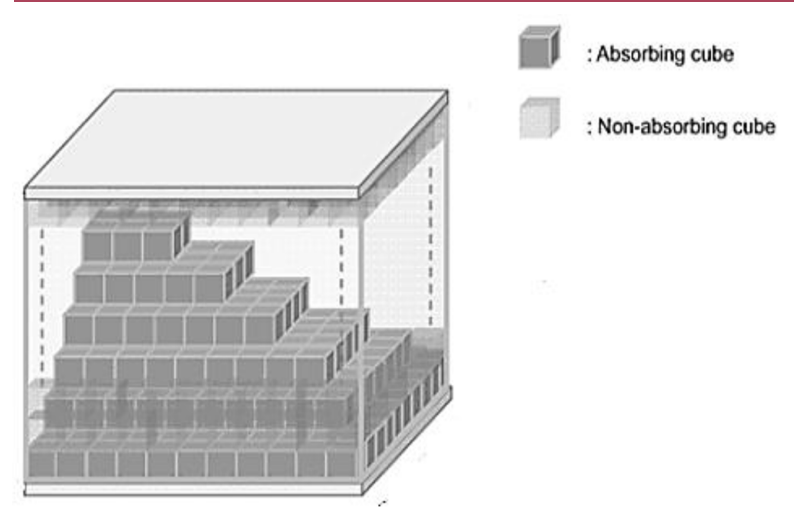

Figure 2: Schematic design of closed stepped wedge form fields "brass cubic blocks with dimension $1 \times 1 \mathrm{~cm}^{3}$ were arranged for making stepped wedge filter and Perspex blocks with dimension $1 \times 1 \mathrm{~cm}^{3}$ were used for filling the empty spaces inside the compensator box made of aluminum".

dosimeter. For block-piled-up compensator and open fields at $10 \mathrm{~cm}$ depth and three energy levels of 6, 10 and $18 \mathrm{MV}$, in fields with dimensions of $15 \times 15 \mathrm{~cm}^{2}$ was performed by the use of Mapcheck 2 dosimeter manufactured by Sun Nuclear Co. of the USA and solid water phantom SP34 manufactured by ScanditronixWellhofer of Germany-Sweden.

\section{Results}

In this study, the data related to the theoretical dose presented by the use of generalized Clarkson's equation were derived and compared with the results obtained from practical measurements, and their findings were demonstrated as charts indicating the error percent calculated and measured values. Generalized Clarkson's equation is:

$\overline{T M R}\left(d, r_{d}\right)=\left[K_{P} \times T \times T M R_{0}+(2 T-1) \times \overline{S M R}\left(d, r_{d}\right)\right] \times \frac{S_{P}(0)}{S_{P}\left(r_{d}\right)}$

As an instance, the results of comparison of theoretical and measured doses at the energies of 6,10 and $18 \mathrm{MV}$ in $10 \mathrm{~cm}$ depth at the field dimensions of $15 \times 15 \mathrm{~cm}^{2}$, with 1,5 $\mathrm{cm}$ thicknesses of flat compensator filters are drawn in Figures 3, 4 and 5, and the results of comparison of theoretical and measured doses in block-piled-up compensator are drawn in Figure 6. These results demonstrate the data derived by the use of generalized Clarkson's method in flat and block-piled-up compensator filters are in good agreement with the data resulted from measurement, the highest error of the proposed equation was 3\% for flat filter and less than 5\% for block-piled-up filter.

\section{Discussion}

IMRT has a modulated intensity in the field and works based on inverse planning. In this technique a pencil beam, convolution superposition algorithms and Monte Carlo method are selected for dose calculation. Monte Carlo method is, however, under investigation due
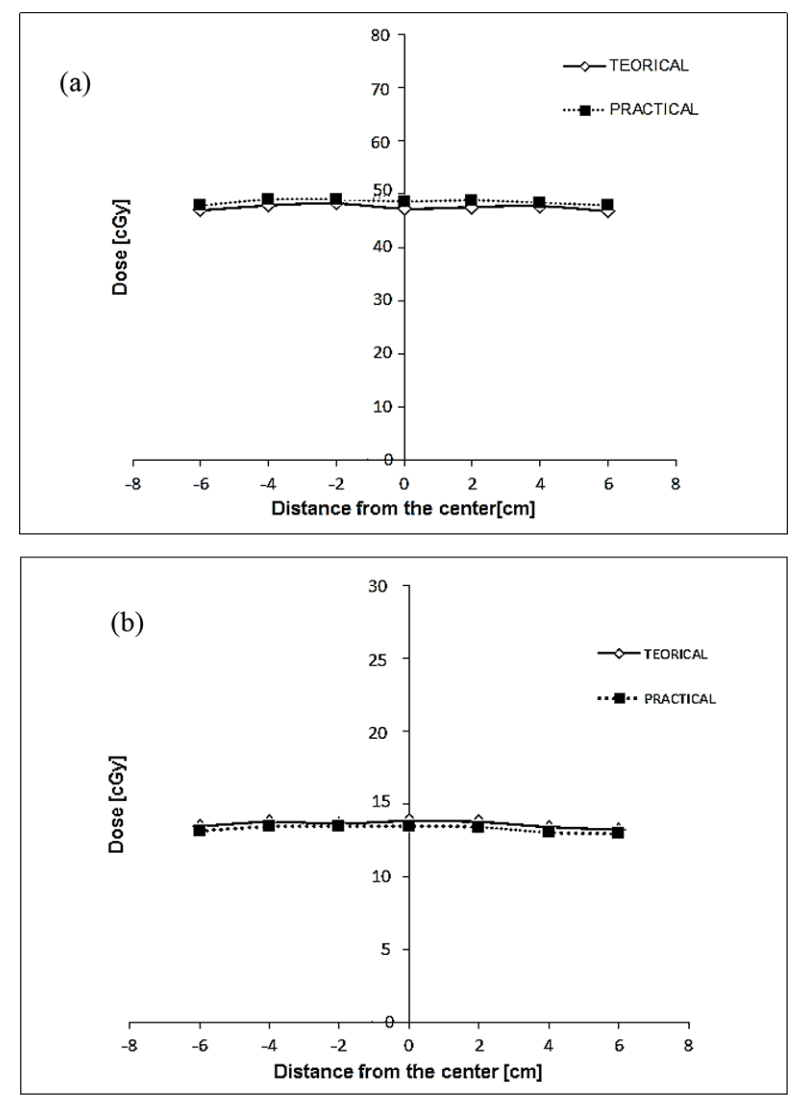

Figure 3: Comparison chart of theoretical and measured doses at $6 \mathrm{MV}$ energy, $10 \mathrm{~cm}$ depth, $15 \times 15 \mathrm{~cm}^{2}$ field dimensions, for (a) 1 $\mathrm{cm}$ thickness, (b) $5 \mathrm{~cm}$ thickness of flat compensator filter. 

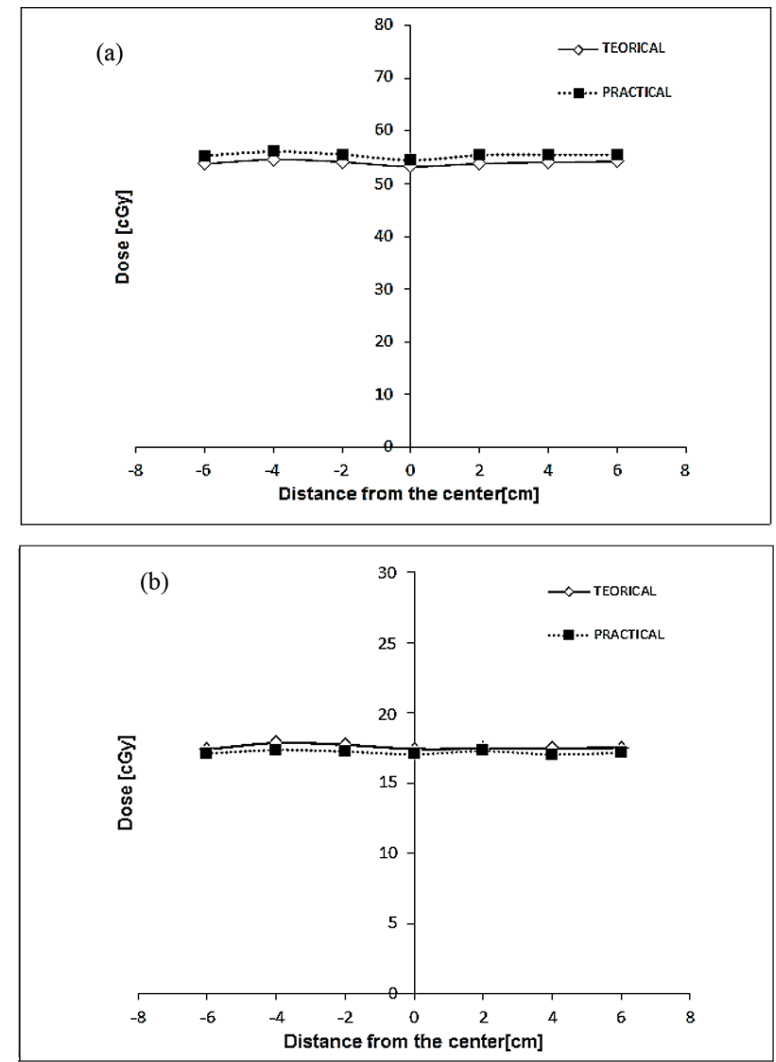

Figure 4: Comparison chart of theoretical and measured doses at $10 \mathrm{MV}$ energy, $10 \mathrm{~cm}$ depth, $15 \times 15 \mathrm{~cm}^{2}$ field dimensions, for (a) 1 $\mathrm{cm}$ thickness, (b) $5 \mathrm{~cm}$ thickness of flat compensator filter.

to limitations of calculation speed. Among existing methods of dose calculation, Clarkson's method is one of the leading methods used for dose calculation at open points present in the irregular fields, part of which has partially been shielded and, factors such as presence of wedge and/or non-uniformity of surface are ignored. By using the method presented in this study, the capability of calculation of dose changes under compensator filters was attained and, in addition, one can point to the application of this method in IMRT quality assurance as well. In the treatment planning software in the form of inverse planning in IMRT, an intensity plan is derived for each field. If, in IMRT, compensator mode is selected, the intensity plan shall be converted to compensator thickness. In the existing intensity plans,
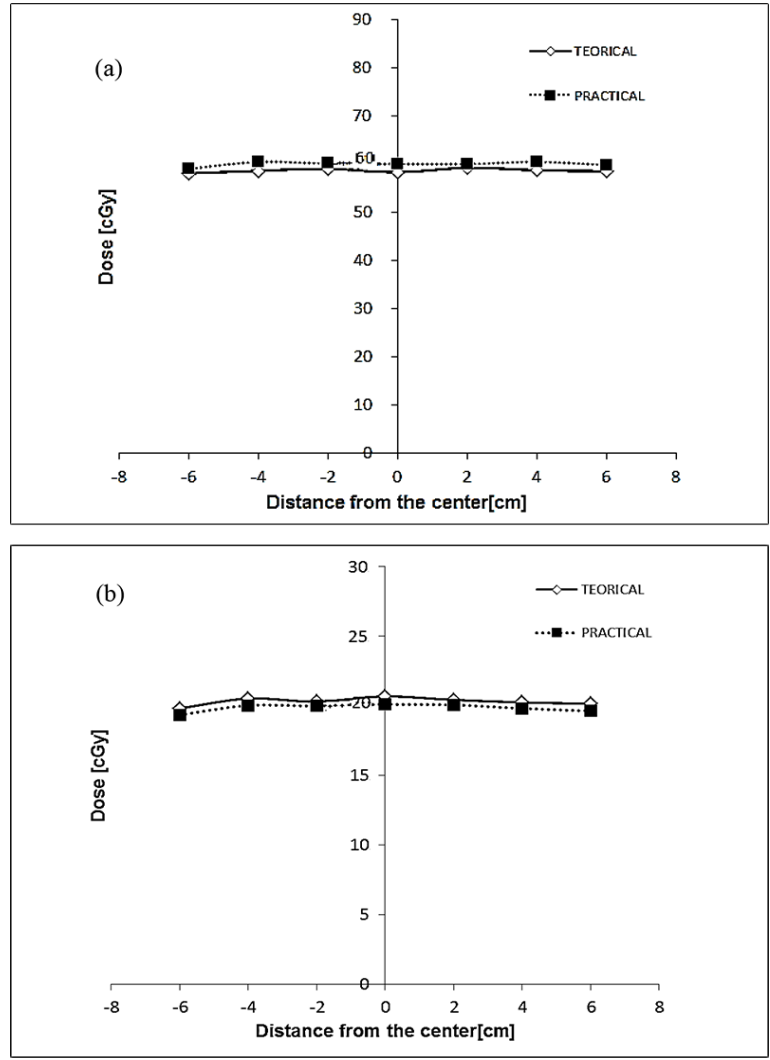

Figure 5: Comparison chart of theoretical and measured doses at $18 \mathrm{MV}$ energy, $10 \mathrm{~cm}$ depth, $15 \times 15 \mathrm{~cm}^{2}$ field dimensions, for (a) 1 $\mathrm{cm}$ thickness, (b) $5 \mathrm{~cm}$ thickness of flat compensator filter.

for each point in intensity plan, the $\mathrm{I} / \mathrm{I}_{0}$ ratio has been obtained by the treatment planning software. Based on $\mathrm{I} / \mathrm{I}_{0}$ ratio for each point, one can obtain the dose of every point by the use of Clarkson's method. In this study, since the purpose is not the calculation of treatment planning error and only the verification of the presented equation is indicated there. Instead of extracting $\mathrm{I} / \mathrm{I}_{0}$ ratio from treatment planning software, Mapcheck2 dosimeter was used. Thus, by using dosimeter, the doses of various points of the field were measured for open fields in the absence of compensator filter. In the presence of the filter, in this way, the $\mathrm{D} / \mathrm{D}_{0}$ ratio, which is equal to $T$, was derived for all points of the field with $1 \mathrm{~cm}$ spatial resolution. In treatment planning system, resolution fluence can be defined equal to $1 \mathrm{~cm}$ for deriving 

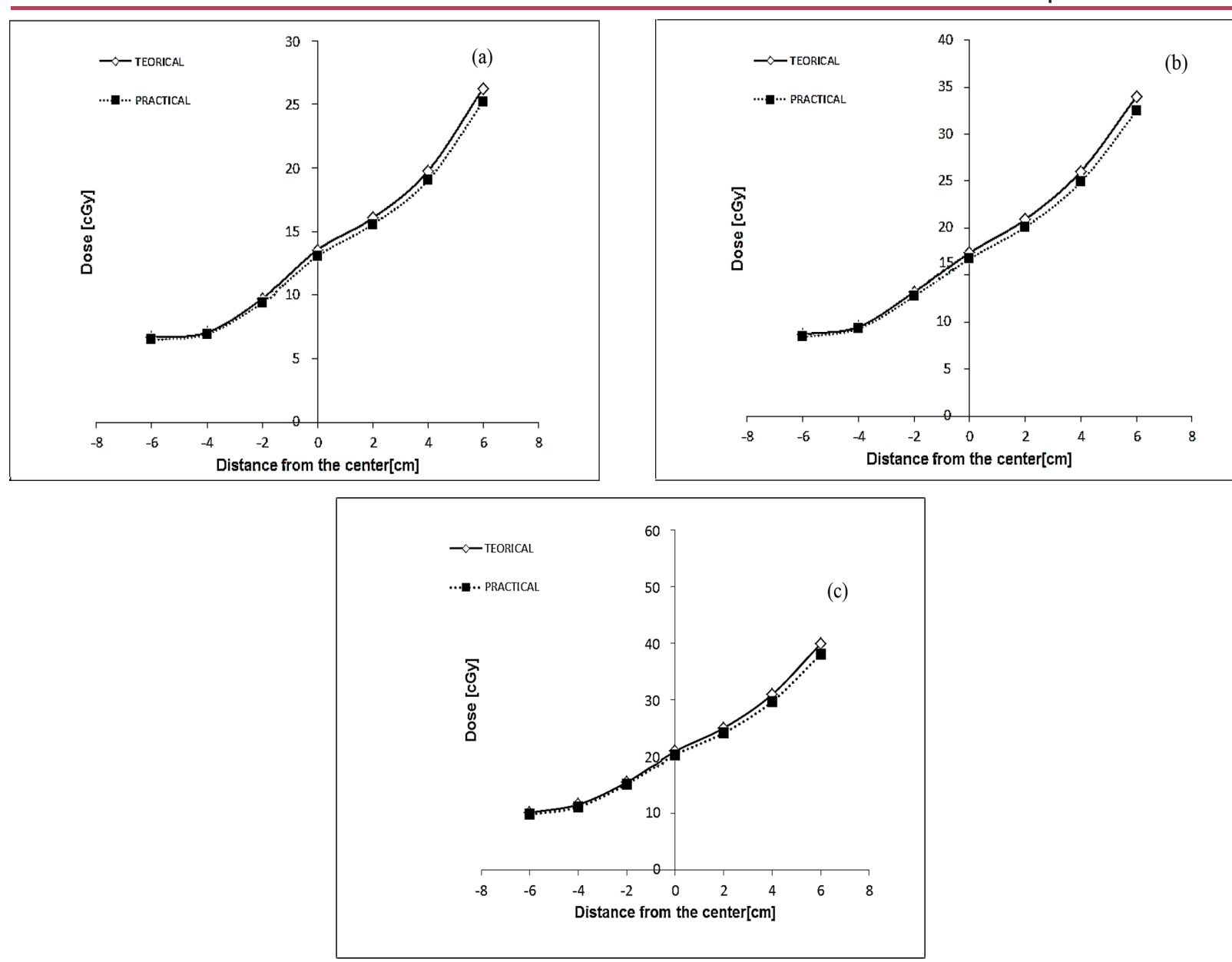

Figure 6: Comparison chart of theoretical and measured doses at $10 \mathrm{~cm}$ depth and $15 \times 15 \mathrm{~cm}^{2}$ field dimensions, at energies of (a) $6 \mathrm{MV}$, (b) $10 \mathrm{MV}$, and (c) $18 \mathrm{MV}$ in block-piled-up compensator filter.

the relative intensity plan, then, the intensity plan is extracted. Several randomly selected points of the field were selected and, by using generalized Clarkson's equation, the theoretical doses of those points were calculated and compared with the measured dose of the same points obtained by dosimeter. The results of calculations revealed that the maximum difference percent between the theoretical and measured doses was 3\% for flat filter and less than 5\% for block-piled-up filter; more errors were observed with block-piled-up filter compared with that of the flat on due to the fact that the existing blocks in the compensator box are arranged alongside each other according to Koji Sasaki's study [19]. Thus, more errors were noted due to the presence of leakage be- tween these blocks. The limitation of the study was the calculation time that can be resolved by software design for the calculations. In our study, efforts were made to eliminate defects existing in other studies to provide the capability for dose calculation at all points under the filter by the use of the presented method.

\section{Conclusion}

In this study, we performed the optimization of dose calculation in IMRT technique based on compensator filter by the use of generalization of common Clarkson's equation. The results of this study demonstrated that the presented equation is reliable and valid, and the proposed equation can be applied for dose calculation at all points under the compensator 
filter or the shielded areas.

\section{Acknowledgment}

This paper is obtained from an M.Sc. project carried out at Kermanshah University of Medical Sciences in close collaboration with Radiotherapy Department of Imam Reza Hospital in Kermanshah, Iran. We would like to express our special thanks to Kermanshah University of Medical Sciences and the Radiotherapy Department of Imam Reza Hospital for providing financial support as well as the clinical and technical assistance required for conducting this research.

\section{Conflict of Interest}

None

\section{References}

1. Cheung K. Intensity modulated radiotherapy: advantages, limitations and future developments. Biomed Imaging Interv J. 2006;2:1-19.

2. Malicki J. The importance of accurate treatment planning, delivery, and dose verification. Reports of Practical Oncology and Radiotherapy. 2012;17(2):63-5.

3. Iqbal K, Isa M, Buzdar SA, Gifford KA, Afzal M. Treatment planning evaluation of sliding window and multiple static segments technique in intensity modulated radiotherapy. Reports of Practical Oncology \& Radiotherapy. 2012;18(2):101-6.

4. Saminathan S, Chandraraj V, Sridhar C, Manickam R. Comparison of individual and composite field analysis using array detector for Intensity Modulated Radiotherapy dose verification. Reports of Practical Oncology \& Radiotherapy. 2012;17(3):15762.

5. Haghparast A, Hashemi B, Eivazi MT. Influence of compensator thickness, field size, and off-axis distance on the effective attenuation coefficient of a cerrobend compensator for intensity-modulated radiation therapy. Med Dosim. 2013;38:259.

6. Papanikolaou N, Battista JJ, Boyer AL, Kappas C, Klein $\mathrm{E}$, Mackie TR, et al. Tissue inhomogeneity corrections for megavoltage photon beams. AAPM Task Group. 2004;65:1-142.

7. Hashimoto S, Karasawa K, Fujita $Y$, Miyashita $H$, Chang W, Kawachi T, et al. Evaluation of beam hardening and photon scatter by brass compen- sator for IMRT. J Radiat Res. 2012;53:999-1005. doi: 10.1093/jrr/rrs048. PubMed PMID: 22915779. PubMed PMCID: PMC3483849.

8. Basran P, Ansbacher W, Field G, Murray B. Evaluation of optimized compensators on a 3D planning system. Med Phys. 1998;25:1837-44.

9. Baka IA, Laub WU, Nusslin F. Compensators for IMRT--an investigation in quality assurance. $Z$ Med Phys. 2001;11:15-22. PubMed PMID: 11487855.

10. Chang SX, Cullip TJ, Deschesne KM, Miller EP, Rosenman JG. Compensators: an alternative IMRT delivery technique. J Appl Clin Med Phys. 2004;5:15-36. PubMed PMID: 15753937. PubMed PMCID: PMC5723484.

11. Linthout N, Verellen D, Van Acker S, Storme G. A simple theoretical verification of monitor unit calculation for intensity modulated beams using dynamic mini-multileaf collimation. Radiother Oncol. 2004;71:235-41.

12. Georg D, Nyholm T, Olofsson J, Kjær-Kristoffersen F, Schnekenburger B, Winkler P, et al. Clinical evaluation of monitor unit software and the application of action levels. Radiother Oncol. 2007;85:306-15.

13. Chang SX, Cullip TJ, Deschesne KM. Intensity modulation delivery techniques:"Step \& shoot" MLC auto?sequence versus the use of a modulator. Med Phys. 2000;27:948-59.

14. Nill S, Tücking T, Münter MW, Oelfke U. Intensity modulated radiation therapy with multileaf collimators of different leaf widths: a comparison of achievable dose distributions. Radiother Oncol. 2005;75:106-11.

15. Korhonen L. Methods for dose calculation and beam characterization in external photon beam radiotherapy. Helsinki University of Technology; 2009.

16. Zhu J, Yin FF, Kim JH. Point dose verification for intensity modulated radiosurgery using Clarkson's method. Med Phys. 2003;30:2218-21.

17. Khan FM. The physics of radiation therapy, 4th ed. Philadelphia: Lippincott Williams \& Wilkins; 2010.

18. Animesh. Advantages of multiple algorithm support in treatment planning system for external beam dose calculations. J Cancer Res Ther. 2005;1:12-20. PubMed PMID: 17998620.

19. Sasaki K, Obata Y. Dosimetric characteristics of a cubic-block-piled compensator for intensity-modulated radiation therapy in the Pinnacle radiotherapy treatment planning system. J Appl Clin Med Phys. 2007:8:85-100. 